\title{
Fatigue Damage Identification and Remaining Useful Life Estimation of Composite Structures using Piezo Wafer Active Transducers
}

\author{
Richard Loendersloot ${ }^{1[0000-0002-1113-8203]}$, Mohammad \\ Ehsani $^{2[0000-0003-2322-4915]}$, and Mahnaz Shamshirsaz ${ }^{2}$ \\ 1 University of Twente, Dynamics Based Maintenance, P.O. Box 217, 7500AE, \\ Enschede, The Netherlands, r.loendersloot@utwente.nl \\ 2 Amirkabir University of Technology, Mechatronics Laboratory, New Technologies \\ Research Center (NTRC), Tehran, Iran
}

\begin{abstract}
The prediction of fatigue damage accumulation is a crucial element in the estimation of the Remaining Useful Life of composite structures subjected to cyclic loading. In this paper, two Glass-Fibre Reinforced Plastics, a thin strip and a thick beam, are subjected to fatigue load while being monitored with Piezo Wafer Active Sensors. Two distinct methods, one based on Electro-Mechanical Impedance Spectroscopy (EMIS) and one based on the Reconstruction Algorithm for Probabilistic Identification of Damage (RAPID), are employed. Both methods are mostly used for damage detection, yet not for damage accumulation monitoring. The results presented in this paper show that damage accumulation can be followed during fatigue loading of the test objects. The trends shown in the damage accumulation graphs give an indication of the damage accumulation, and even a change in the damage evolution stage, yet a complete RUL estimation is not possible without further analysis of the experiments, possibly assisted by numerical modelling.
\end{abstract}

Keywords: Electromechanical Impedance · Piezo Wafer Active Sensors · Fatigue · Damage Accumulation · GFRP · RUL · SHM

\section{Introduction}

Composite structures used in aerospace industry or wind turbine blades are usually exposed to dynamic loading such as repeated impact and large amplitude vibration that induces fatigue damage as a critical safety issue in their design and maintenance. Acousto-Ultrasonic (AU) monitoring with integrated Piezo Wafer Active Sensors has proven its merits in damage identification in composite materials, be it primarily on impact-induced delaminations in thin composite structures [1-3]. Recently, the potential of this technique to follow the fatigue damage accumulation has been proven. Loendersloot et al. [4] investigated the capability of Reconstruction Algorithm for Probabilistic Inspection of Damage (RAPID) for early damage detection in a thick composite beam. 
Electro-Mechanical Impedance Spectroscopy (EMIS) is another active health monitoring technique for damage identification [5]. This method takes advantage of simultaneous actuating and sensing properties of PWAS to diagnose the host structure deviation from its pristine state $[6,7]$. EMIS has also shown its potential for fatigue damage accumulation monitoring in homogeneous and composite structures. Yu et al. [8] studied the effectiveness of EMIS technique for fatigue damage identification in GFRP and distinguished between sensor/actuator system and host structure degradation. Annamdas et al. [9] reported a correlation between fatigue damage initiation and evolution stages and shifting of real of admittance spectrum due cyclic loading of structure, which was further investigated by Soh et al. [10].

Giurgiutiu et al. [11] pointed at the complementarity of AU and EMIS in terms the sensitivities to the far field and near field damage. Cherrier et al. [12] utilized Inverse Distance Weighting (IDW) index obtained from exponential attenuation of $\mathrm{AU}$ waves to localize damage in CFRP composite structures using a damage indicator computed from EMIS.

A step yet to make, is the prognosis of the evolution of damage accumulation. This will allow for an estimation of the Remaining Useful Life (RUL) based on actual measured damage accumulation, rather than on a load history, which is limited by uncertainties on the damage initiation and propagation [13].

The main purpose of this study is to examine the capability of AU and EMIS towards early fatigue damage monitoring of composite structures. Furthermore, feasibility of using the damage accumulation for an estimation of the Remaining Useful Life (RUL) is addressed. Three point bending fatigue tests was conducted on both a thin and a thick GFRP composite structures and the health level of the specimens were evaluated by intermittent inspection via propagating (AU) and standing (EMIS) waves signature.

Extensive research has been carried out around effective fatigue damage mechanisms in composite materials $[14,15]$. Cumulative damage caused by cyclic loading of composite materials is a multi-state degradation process [16]. As the cyclic loading continues, the density of matrix micro-cracks increases up to a saturation state, followed by a coalescence of matrix micro-cracks and fibre-matrix debonding. In composites made of tough matrices, this step reveals as excessive deformation of matrix, which in turn results in localized failure of fibres. Fatigue damage evolution in the form of matrix micro-cracks as well as fibrematrix debonding leads to delamination of adjacent layers. Ultimately, damage progresses up to the final failure phase of the composite structure, as it loses its load bearing capacity. The precise ordering of different steps from microcrack to failure depends on laminate lay-up, the constituents of the composite, manufacturing defects, the loading profile and environmental conditions [17].

The presence of micro-cracks generally does not yet affect the global forcedisplacement relation, which is only affected once the structural integrity is compromised significantly due to debonding, fibre breakage or delaminations. The main concept of this research is that the micro-cracks do affect the local response 
to Acousto-Ultrasonic waves travelling through the structure. Hence, capturing the changes in this response, will allow for early fatigue damage identification.

\section{Active SHM methods}

Two methods, both relying on the simultaneous actuating and sensing capabilities, are used in this research: Electro-Mechanical Impedance Spectroscopy (EMIS) and the Reconstruction Algorithm for Probabilistic Inspection of Damage (RAPID).

\subsection{Electro-mechanical impedance spectroscopy (EMIS)}

Electro-Mechanical Impedance Spectroscopy (EMIS) is classified as a high frequency vibration based active damage detection technique in SHM. The basis of damage detection in EMIS is the damage induced alteration of mechanical impedance, which is a combination of mass, stiffness, damping and boundary conditions effects and thus dynamic behaviour of host structure. The function of piezoelectric patch coupled with the host structure is to convert these changes to electromechanical impedance spectrum modification in the form of peak frequency and amplitude shifting. According to previous studies, the real part of complex electromechanical impedance is more sensitive to host structure defects, while the imaginary part is more influenced by bonding layer degradation [18].

The most common damage indicators for damage quantification with EMIS are the Root Mean Square Deviation (RMSD), the Mean Absolute Percentage Deviation (MAPD) and the Correlation Coefficient Deviation (CCD). These are defined as follows:

$$
\begin{aligned}
R M S D & =\sum_{k=\omega_{I}}^{\omega_{F}} \sqrt{\frac{\left(Z_{E, H}(k)-Z_{E, D}(k)\right)^{2}}{Z_{E, H}^{2}(k)}} \\
M A P D & =\frac{1}{N} \sum_{k=\omega_{I}}^{\omega_{F}} \frac{\left(Z_{E, H}(k)-Z_{E, D}(k)\right)}{Z_{E, H}(k)} \\
C C D & =1-\frac{\sum_{k=\omega_{I}}^{\omega_{F}}\left(Z_{E, H}(k)-\bar{Z}_{E, H}(k)\right)\left(Z_{E, D}(k)-\bar{Z}_{E, D}(k)\right)}{\sqrt{\sum_{k=\omega_{I}}^{\omega_{F}}\left(Z_{E, H}(k)-\bar{Z}_{E, H}(k)\right)^{2}} \sqrt{\sum_{k=\omega_{I}}^{\omega_{F}}\left(Z_{E, D}(k)-\bar{Z}_{E, D}(k)\right)^{2}}}
\end{aligned}
$$

Here, $Z_{E, H}$ and $Z_{E, D}$ represent the real part of healthy and damaged impedance signature; $\bar{Z}_{E, H}$ and $\bar{Z}_{E, D}$ stand for their mean; $\omega_{I}$ and $\omega_{F}$ are the intended frequency range limits. The frequency range is selected according to its sensitivity to any particular damage. 


\subsection{Reconstruction Algorithm for Probabilistic Inspection of Damage (RAPID)}

The Reconstruction Algorithm for Probabilistic Inspection of Damage (RAPID) algorithm is based on the comparison of the time signals of each of the actuatorsensor paths in pristine and post-damage state. These differences are condensed to the damage index $\rho$. Typically, the correlation coefficient is used [19,1], although a range of methods is available [20,2]. For the application of the thick composite beam, a slightly modified form of the Signal Amplitude Squared Percentage difference algorithm (SAPS), was proven to be most effective [21]:

$$
\rho_{\mathrm{SAPS}, k}=1-\left(\frac{\max \left(S_{H, k}\right)-\max \left(S_{D}\left(t_{\max }^{H}-\Delta t: t_{\max }^{H}+\Delta t\right)\right)}{\max \left(S_{H, k}\right)}\right)^{2}
$$

Where $S_{D}$ refers to the current signal and $S_{H}$ to the reference signal. $\Delta t$ refers to the small time range around the time $t_{\max }^{H}$ at which the maximum peak is observed in $S_{H}$. The index $k$ refers to the actuator-sensor pair.

Subsequently, a probability function is used that indicates the probability that an anomaly at location $(x, y)$ has caused the difference between the signals $S_{H}$ and $S_{D}$. It uses a geometrical function $R(x, y)$, specifying the geometrical distance from point $(x, y)$ to the direct line between the two transducers $i$ and $j$, ceiled by the threshold value $\beta$ :

$$
\begin{aligned}
& R(x, y)= \\
& \begin{cases}\frac{\sqrt{\sum_{\xi=x, y}\left(\Delta \xi_{i}+\Delta \xi_{i j}\right)^{2}}+\sqrt{\sum_{\xi=x, y}\left(\Delta \xi_{j}-\Delta \xi_{i j}\right)^{2}}}{(1-2 \alpha) \sqrt{\Delta x_{i j}^{2}+\Delta y_{i j}^{2}}} & \text { for } R(x, y)<\beta \\
\beta & \text { for } R(x, y) \geq \beta\end{cases} \\
& \Delta x_{k}=\alpha\left(x-x_{k}\right), \quad \Delta y_{k}=\alpha\left(y-y_{k}\right) \quad \text { with } k=i, j ; \quad \Delta x_{i j}=x_{i}-x_{j}
\end{aligned}
$$

where $\left(x_{i}, y_{i}\right)$ and $\left(x_{j}, y_{j}\right)$ indicate the locations of transducer $i$ and $j$ respectively. Following Moix-Bonet et al. [22] $\alpha$ and $\beta$ are obtained from an optimisation process. Overlaying all path results gives a damage intensity probability map. The damage intensity probability $I$ at an arbitrary position $(x, y)$ is given by:

$$
I(x, y)=\sum_{k=1}^{N_{p}}\left(\left(1-\rho_{k}\right)\left(\frac{\beta-R(x, y)}{\beta-1}\right)\right)
$$

with $\rho_{k}$ being the damage indicator of the $k^{\text {th }}$ actuator-sensor path, $N_{p}$ the number of paths.

\section{$3 \quad$ Experimental procedure}

Two different specimen were subjected to a three-point bending fatigue test. Both test objects are made of glass-fibre reinforce plastic, but one is relatively 
thin, while the other is relatively thick. Both are equipped with PWAS transducers, yet another type of use (EMI versus RAPID) is used.

\subsection{Material and specimen preparation}

For the EMIS test, 7 layer angle-ply $\left( \pm 55^{\circ}\right)$ unidirectional E-glass fiber fabrics (EC 9-68) with an area density of $425 \mathrm{gr} / \mathrm{m}^{2}$ and layer thickness of $0.45 \mathrm{~mm}$ were used to fabricate the specimens. The low-viscosity resin, F type Bisphenol mixed with the hardener Sika CH83-2 in a ratio of 10:1 was used for impregnation (vacuum-infusion) of the fabrics. Rectangular specimens (see Fig. 1) with average dimensions of $l \times b \times h=250 \times 25 \times 2.5 \mathrm{~mm}^{3}$ were cut out of laminates of $400 \times 400 \times 2.5 \mathrm{~mm}^{3}$ by a water-jet cutting machine according to ASTM D3039 [23] and ASTM D7791-12 [24]. The fatigue specimens were instrumented with four PWAS, two in the section under loading and the other two out of loading area.

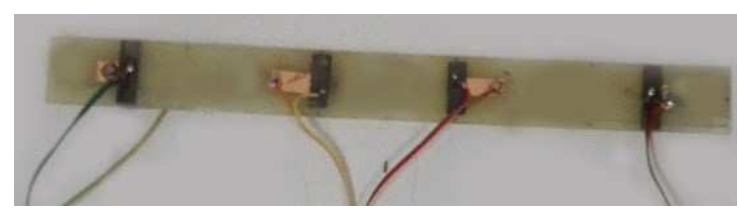

Fig. 1. Test specimen for the EMIS test.

The uni-directional, 96 layer non-crimp glass fiber fabric reinforced plastic (Hexion RIM 135) beam $\left(l \times b \times h=900 \times 60 \times 56 \mathrm{~mm}^{3}\right)$, was manufactured by WMC. It was instrumented by the University of Twente with eight PiCeramic DuraAct PZT transducers, four on each side, as shown in Fig. 2.

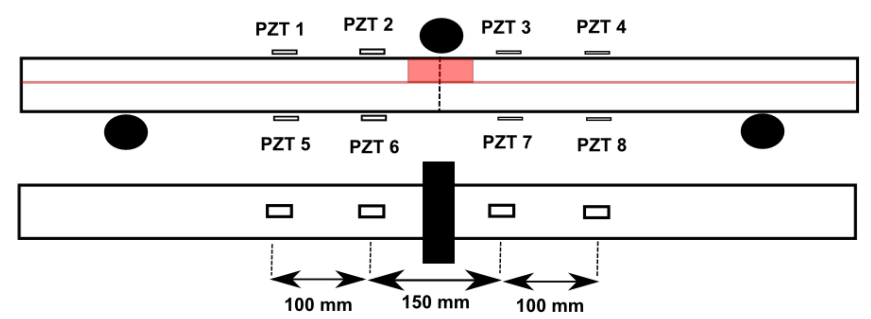

Fig. 2. Schematic of the PWAS instrumented glass fibre reinforced beam. Black circles: the plunger (top one) and the two supports of the three point-bending set-up. 


\subsection{Experimental set-up and instrumentation}

The bending fatigue loading on the thin strip was applied in a displacement control scheme with a frequency of $3 \mathrm{~Hz}$. The load was kept well below the tensile strength limit of the material and the durability threshold of the adhesive layer.

The cyclic loading was interrupted at pre-specified cycles up to 37,500 cycles. Next, the specimen was suspended and each PWAS was actuated in turn with a chirp signal generated by Agilent 33220A arbitrary waveform generator. At the same time, the PicoScope 4424 was used to record the steady state response of the system in the form of complex electromechanical impedance. In order to select the optimal frequency range, a large domain was swept experimentally and qualitative evaluation of the acquired spectrum, resulted in 10 to $60 \mathrm{kHz}$ as the range sensitive to fatigue damage.

A similar approach was followed for the fatigue test of the thick composite: a frequency of $2 \mathrm{~Hz}$ was used and the test was interrupted at pre-defined intervals, shortening with increasing number of total cycles, to allow the acousto-ultrasonic measurements to be executed. The beam failed after nearly 2.7 million cycles, after 2183 measurement series. Data was acquired with a NI PXI DAQ system, with a relay unit. An external function generator and signal amplifier were used to activate the PWAS assigned as actuator. The excitation signal is a short, Hanning windowed burst signal of 3.5 cycles at a fixed frequency. Each test was repeated with excitation frequencies ranging from $40-340 \mathrm{kHz}$ in steps of $20 \mathrm{kHz}$.

\section{Results \& Discussion}

\subsection{Thin GFRP Strip and EMIS}

The EMI spectrum of the four transducers are shown in Fig. 3 for different numbers of loading cycles. The larger amplitude of transducers 2 and 3 compared to that of 1 and 4 is attributed to their proximity to the degraded zone. A constant vertical offset over the full spectrum is observed, implying the shape of the spectrum does not show severe changes, which suggest that the composite structure is in the initial stage of fatigue damage (matrix cracking) and there is no transition to the secondary phase of fibre-matrix de-bonding or delamination.

After de-trending, by correcting for the vertical shift, a decrease of the peak amplitudes of the EMI spectrum is observed, as seen in the close-up view in Fig. 4. This indicates an alteration in the strip's structural damping and suggests the evolution of fatigue damage, in agreement with the results of $\mathrm{Yu}$ et al. [8]. Amplitude decrease is attributed to an increase in the length of initial micro-cracks and also the density of matrix micro-cracks and thus an increase in the friction surfaces available in the specimen which result in more vibrational energy loss, especially in high frequency excitations. No frequency shifts are observed in the impedance graphs. Frequency shifts are generally attributed to stiffness degradation. Hence, it can be concluded that the global stiffness of the 

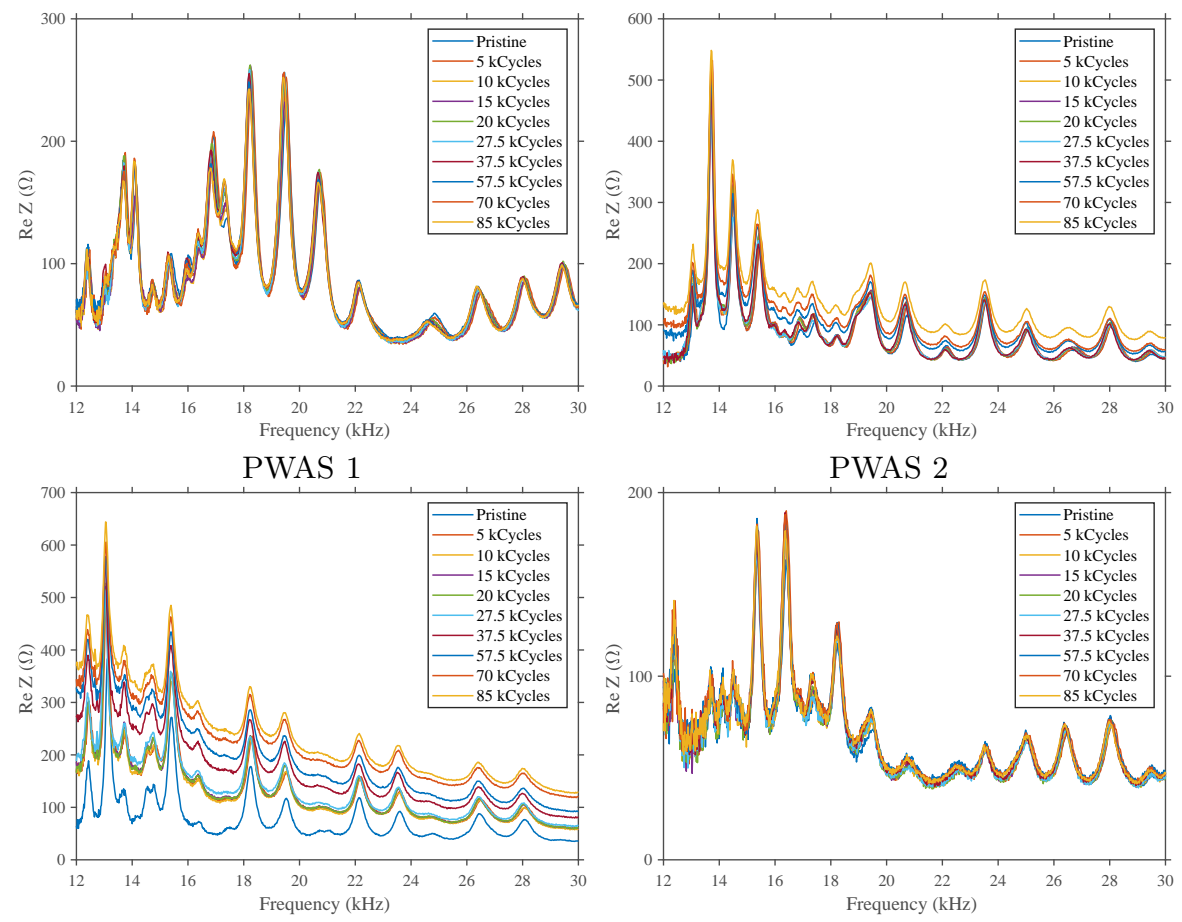

PWAS 3

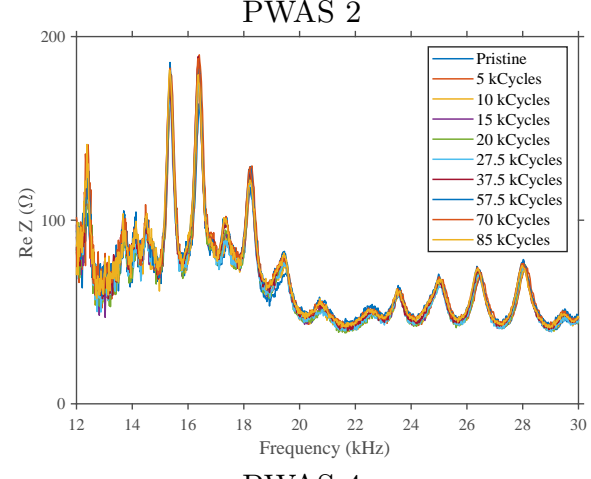

PWAS 4

Fig. 3. EMI spectrum versus the number of fatigue cycles.

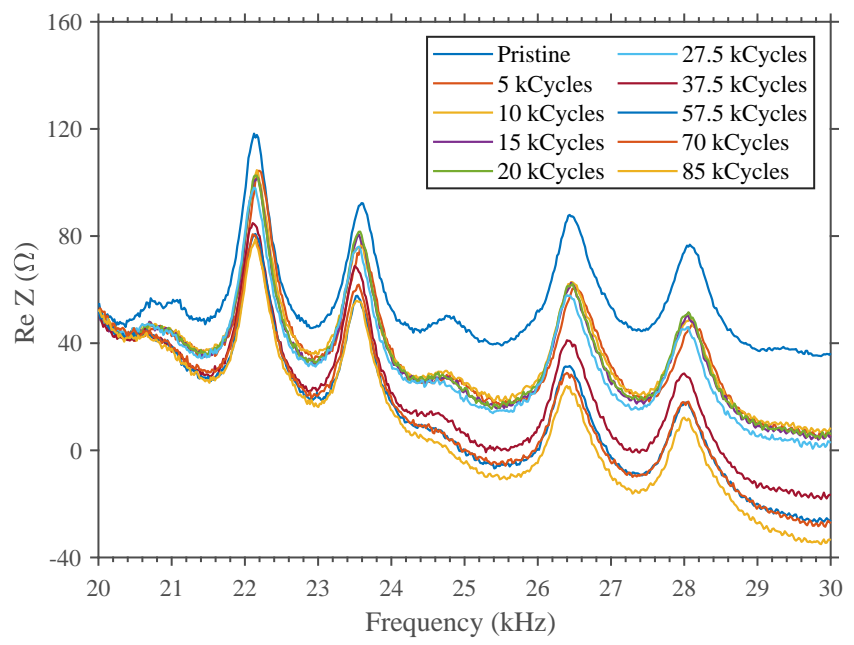

Fig. 4. Close-up view of EMI spectrum vs. the number of fatigue cycles captured by transducer 3 (20 to $25.5 \mathrm{kHz}$ ). 
strip seems to be unaffected by the accumulating fatigue damage during these measurements.

Fig. 5 depicts the RMSD, CCDM and MAPD damage metrics variation for transducer 2 and 3 . The magnitude of acquired DIs from transducers 2 and 3 is several times larger (25 times greater for PZT 3 ) than that of transducers 1 and 4 , due to the proximity of the damage. The results show that the CCDM algorithms is insensitive to the accumulation of damage, while the RMSD and MAPD methods are. Typically, CCDM is more influenced by reshaping and horizontal shifts. However, the early stages of fatigue damage evolution have the greatest impact on the structural damping, rather than on the stiffness. The RMSD and MAPD indices are more effective metrics for early fatigue damage monitoring in composite structures.

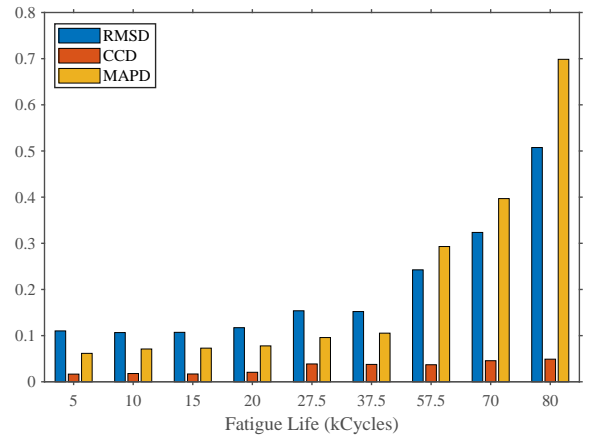

PWAS 2

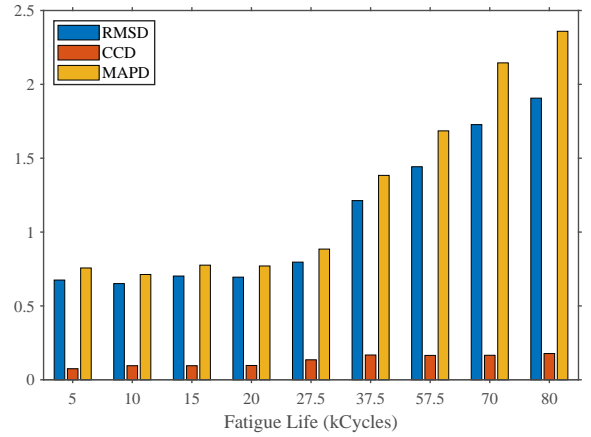

PWAS 3

Fig. 5. Evolution of Damage Indices based on RSMD, CCDM and MAPD versus number of fatigue cycles.

Both RMSD and MAPD show a progressively increasing trend for an increasing number of fatigue cycles. The change of slope can be used as a threshold for critical damage accumulation, yet the physical interpretation of this limit cannot be retrieved from the experimental data. Numerical models can assist in the interpretation and improve the applicability for RUL estimations.

\subsection{Thick GFRP Beam and RAPID}

Typically, the results of the RAPID measurements as applied in the fatigue test of the thick composite beam are condensed to damage intensity probability maps. The time signals of the waves travelling from one transducer to another after a number of cycles are compared to the time signal obtained prior to the fatigue test. The change in time signals is quantified here using the SAPS algorithm and then combined in a spatial distribution of the probability of damage [4]. The resulting damage intensity probability graphs after 87,000 and $2,476,000$ cycles are shown in Fig. 6 . The signal actuation frequency was $200 \mathrm{kHz}$ in this case. 

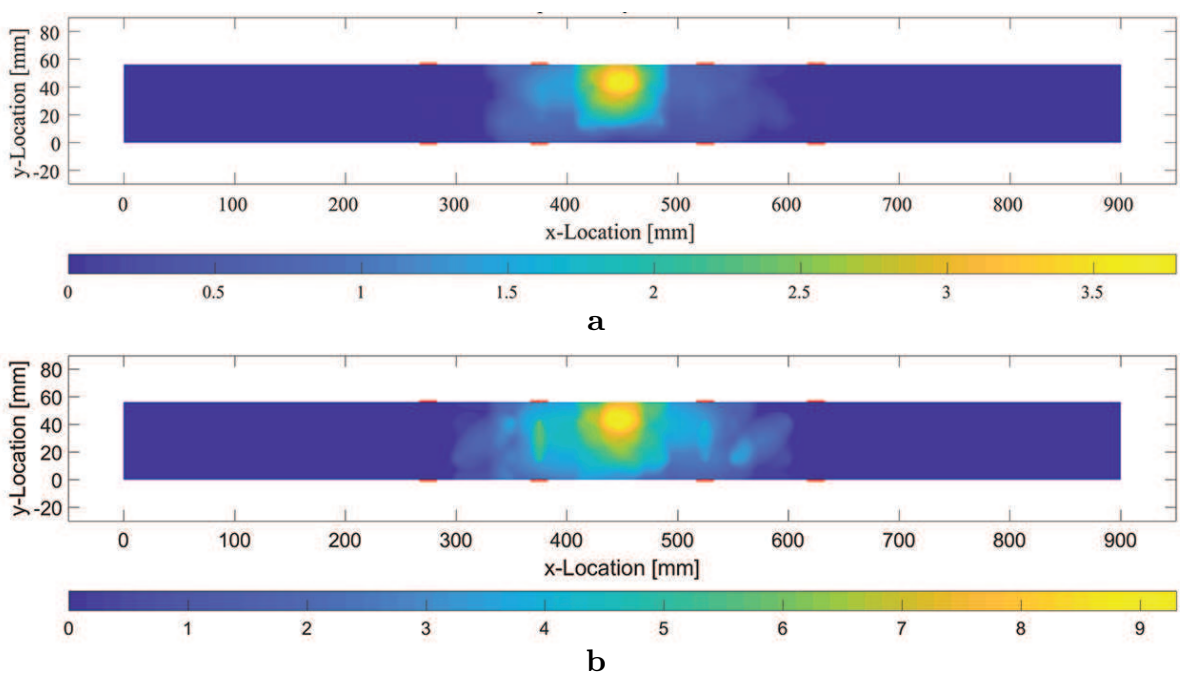

Fig. 6. The damage probability (RAPID) maps, based on the SAPS algorithm and an actuation frequency of $2000 \mathrm{kHz}$, after a: 87,000 cycles; and b: 2,476,000 cycles. The red lines indicate the location of the transducers

Normally, the probability plots are used to identify the presence of a (delamination) damage, but here, the change of these graphs over time is of interest, as it can be used to follow the accumulation of fatigue damage in the structure. A measure needs to be defined to quantify the amount of accumulated damage in the probability graphs. It is worth noting that the value of the damage intensity probability has no direct physical meaning, as it is based on the maximum amplitudes found in the time signals of the waveforms. The most straightforward approach is to take the maximum value of the probability plots, which results in the red line in Fig. 7. In this case, no noise correction was applied on the time signals, in contrast to an earlier analysis [4], resulting in a number of outliers and some noise. However, the general trend of the line is maintained: a sharp increase in the beginning, attributed to some settling and initial damage at the start of the measurements, followed by a slow increase up to approximately 2,000,000 cycles. The small decrease is attributed to noise, as it disappears after the noise correction [4]. The last phase is characterised by a slightly higher probability value, yet a firm conclusion is hard to state based on these results. Hence, it is difficult to set a threshold for a certain damage accumulation limit after which accelerated damage growth and a nearing end of life is expected.

Although it is already a significant improvement compared to force-displacement based damage accumulation estimations - as they only issue a very late warning of the approaching end of life - it is attempted to improve the predictive capabilities of this method by using another feature of the probability maps. The correlation coefficient, based on the comparison between the probability map at a certain cycle number and the first probability map after 2,000 cycles, is considered as a good candidate. It takes in to account the overall shape of the 


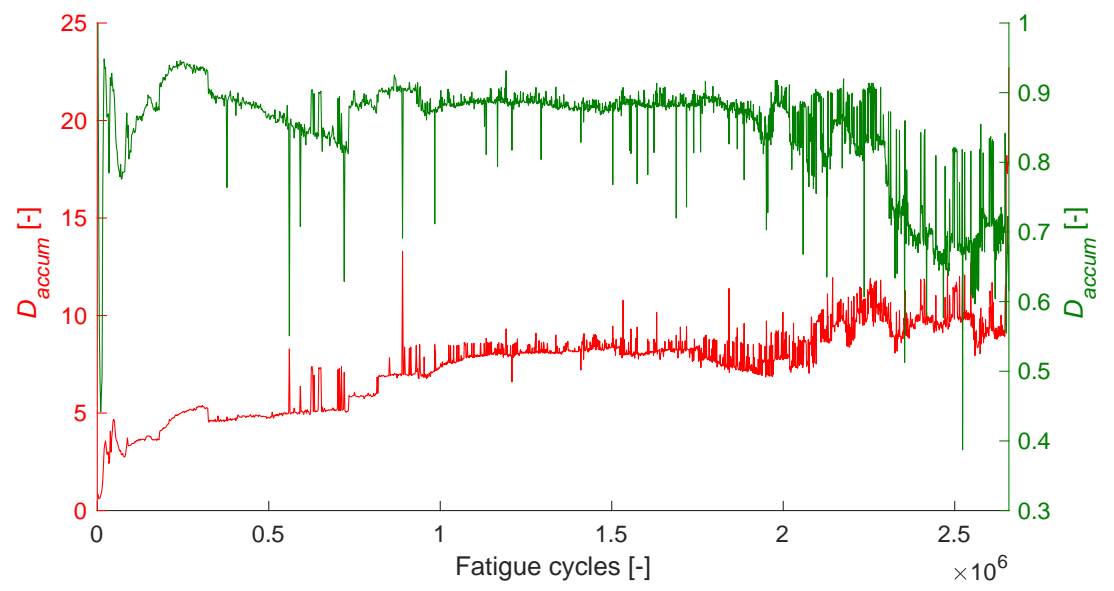

Fig. 7. Damage accumulation show by the evolution of the maximum value of the probability maps (red line) and correlation coefficient (green line) for increasing number of fatigue cycles, for an actuation frequency of $200, \mathrm{kHz}$.

affected regions and thus provides a much more global comparison compared to the maximum value. The resulting correlation coefficient is shown as the green line in Fig. 7. Apart from some spikes, which are believed to be associated with measurement inaccuracies and noise, rather than with actual damage accumulation, a fairly constant correlation coefficient is observed up to just over 2,000,000 cycles. A sudden drop is observed close to 2,300,000 cycles, indicating the damage has proliferated to other regions, or new damage regions are initiated. The jump corresponds reasonable well with the increase in the probability value, but is more conclusive. Moreover, it agrees with the expected step-wise damage evolution as described in the introduction of this paper and in the work of Talreja and Singh [17].

It is believed that further signal processing, including noise corrections, will improve the capabilities of the correlation coefficient, and possibly other methods that assess the global changes of the probability maps, in terms of early damage identification. Numerical models may further assist in improving the predictive capabilities of this method.

\section{Conclusions}

Comparison of the results of two fatigue tests, on two different types of test objects, both equipped with PWAS transducers, and using two different approaches for the use and data analysis, revealed that:

- The versatile nature of PWAS transducers make them ideal for application in a wide range of structures;

- Both types of analyses investigated (EMIS and RAPID) allow for a damage accumulation estimation throughout the fatigue life of the composites, 
that outperforms existing, and commonly applied, force-displacement based mathods; and

- In both cases, the support of numerical methods has the potential to stronger link the physics of damage accumulation with the measured features in the signals.

\section{Ackowledgement}

The work presented is funded by the Dutch TKI Wind at Sea project SLOWIND, grant number TEWZ 115012. This support is gratefully acknowledged by the authors.

\section{References}

1. Z. Su and L. Ye. Identification of Damage Using Lamb Waves From Fundamentals to Applications, volume 48 of Lecture Notes in Applied and Computational Mechanics. Springer, 2009.

2. R. Loendersloot, I. Buethe, P. Michaelides, M. Moix Bonet, and G. Lampeas. Smart Intelligent Aircraft Structures (SARISTU): proceedings of the final project conference, chapter Damage Identification in Composite Panels - Methodology and Visualisation, pages 579-604. Springer, 2015.

3. M. Moix Bonet, P. Wierach, R. Loendersloot, and M. Bach. Smart Intelligent Aircraft Structures (SARISTU): proceedings of the final project conference, chapter Damage Assessment in Composite Structures Based on Acousto-Ultrasonics Evaluation of Performance, pages 617-629. Springer, 2015.

4. R. Loendersloot, M. Venterink, A. Krause, and F. Lahuerta. Acousto-ultrasonic damage monitoring in a thick composite beam for wind turbine applications. In Proceeding of the 9th European Workshop on Structural Health Monitoring, 2018.

5. N. Sepehry, F. Bakhtiari-Nejad, M. Shamshirsaz, and W. Zhu. Nonlinear modeling of cracked beams for impedance based structural health monitoring. In Proceedings of the ASME 2017 International Mechanical Engineering Congress and Exposition, page 8 pages, 2017.

6. N. Sepehry, F. Bakhtiari-Nejad, and M. Shamshirsaz. Thermo-electro-mechanical impedance based structural health monitoring of plates. Composite Structures, 116:147-164, 2014.

7. N. Sepehry, M. Shamshirsaz, and F. Bakhtiari Nejad. Lowcost simulation using model order reduction in structural health monitoring: Application of balanced proper orthogonal decomposition. Structural Control and Health Monitoring, 24(11):e1994, 2017.

8. L. Yu, M. Gresil, P. Pollock, and M. Sutton. Progressive damage detection/diagnosis on composite using electromechanical impedance spectroscopy. In Proceedings of the ASME 2011 International Mechanical Engineering Congress and Exposition, pages 255-262, 2011.

9. V.G.M. Annamdas, Y.Chew, J.H.L. Pang, H.J. Hoh, K. Zhou, and B. Song. Fatigue growth analysis of pre induced surface defects using piezoelectric wafer based impedance method and digital image correlation system. Journal of Nondestructive Evaluation, 33(3):413-426, 2014. 
10. C. K. Soh and Y. Y. Lim. Fatigue damage diagnosis and prognosis using electromechanical impedance technique. Elsevier Ltd, 2016.

11. V. Giurgiutiu, A. Zagrai, and J. Jing Bao. Piezoelectric wafer embedded active sensors for aging aircraft structural health monitoring. Structural Health Monitoring, 1(1):41-61, 2002.

12. O. Cherrier, P. Selva, V. Pommier-Budinger, F. Lachaud, and J. Morlier. Damage localization map using electromechanical impedance spectrums and inverse distance weighting interpolation: Experimental validation on thin composite structures. Structural Health Monitoring, 12(4):311-324, 2013.

13. M.M. Derriso, M.P. DeSimio, Ch.D. McCurry, Ch.M. Schubert Kabban, and S.E. Olson. Industrial age non-destructiveevaluation to information agestructural health monitoring. Structural Health Monitoring, 13(6):591-600, 2014.

14. S.W. Case and K.L. Reifsnider. Fatigue of composite materials. Comprehensive Structural Integrity, 4:405-441, 2007.

15. M. Chiachio, J. Chiachio, A. Saxena, and K. Goebel. An energy-based prognostic framework to predict evolution of damage in composite materials. Structural Health Monitoring (SHM) in Aerospace Structures, pages 447-477, 2016.

16. N. Eleftheroglou and T. Loutas. Fatigue damage diagnostics and prognostics of composites utilizing structural health monitoring data and stochastic processes. Structural Health Monitoring, 15(4):473-488, 2016.

17. R. Talreja and C. V. Singh. Damage and failure of composite materials. Cambridge University Press, 2012.

18. I. Mueller. Inspection of piezoelectric transducers used for structural health monitoring systems. PhD-thesis, University of Siegen, 2017.

19. Z. Su, L. Ye, and Y. Lu. Guided lamb waves for identification of damage in composite structures: A review. Journal of Sound and Vibration, 295(3-5):753780,2006 .

20. Z. Wu, K. Liu, Y. Wang, and Y. Zheng. Validation and evaluation of damage identification using probability-based diagnostic imaging on a stiffened composite panel. Journal of Intelligent Material Systems and Structures, 26(16):2181-2195, 2014.

21. M. Venterink, R. Loendersloot, and T. Tinga. The detection of fatigue damage accumulation in a thick composite beam using acousto ultrasonics. In Proceedings of the first HEAMES conference, London, UK, page 10 pages, 2018.

22. M. Moix Bonet, B. Eckstein, R. Loendersloot, and P. Wierach. Identification of barely visible impact damages on a stiffened composite panel with a probabilitybased approach. In F.K. Chang and A. Guemes, editors, Proceedings of International Workshop on Structural Health Monitoring, page 8 pages. Lancaster, Pennsylvania: DEStech Publications, 2015.

23. ASTM D3039/D3039M-14. Standard test method for tensile properties of polymer matrix composite, 2014.

24. ASTM D777417. Standard test method for flexural fatigue properties of polymer matrix composite, 2017. 